(C2020. This manuscript version is made available under the CC-BY-NC-ND 4.0 license http:// creativecommons.org/licenses/by-nc-nd/4.0/

\title{
Cytotoxic effects of Ridolfia segetum (L.) Moris phytoproducts in cancer cells
}

Ellie Beeby ${ }^{a \sharp}$, Mariana Magalhães ${ }^{\mathrm{b}, \mathrm{c}}$, Marco F.L. Lemos ${ }^{\mathrm{d}}$, Isabel M. Pires ${ }^{\mathrm{a}^{*}}$, Célia Cabral ${ }^{\mathrm{b}, \mathrm{c}, \mathrm{e}^{*}}$

\section{Affiliations}

${ }^{a}$ Department of Biomedical Sciences, Faculty of Health Sciences, University of Hull, HU6 7RX, UK

${ }^{b}$ University of Coimbra, Coimbra Institute for Clinical and Biomedical Research (iCBR), Clinic Academic

Center of Coimbra (CACC), Faculty of Medicine, 3000-548 Coimbra, Portugal

'University of Coimbra, Center for Innovative Biomedicine and Biotechnology (CIBB), 3000-548

Coimbra, Portugal

${ }^{\mathrm{d} M A R E}$ - Marine and Environmental Sciences Centre, Instituto Politécnico de Leiria, ESTM, 2520-630

Peniche, Portugal

${ }^{e}$ Centre for Functional Ecology, Department of Life Sciences, University of Coimbra, Calçada Martim de Freitas, 3000-456 Coimbra, Portugal

${ }^{\#}$ These authors contributed equally.

\section{*Corresponding authors:}

Célia Cabral

Faculty of Medicine, University of Coimbra, Pólo das Ciências da Saúde, Azinhaga de Santa Comba, 3000-

548 Coimbra, Portugal

Tel.: +351239857700

E-mail address: celia.cabral@.fmed.uc.pt

Isabel M. Pires

University of Hull, Department of Biomedical Sciences, Hardy Building, Cottingham Road, Hull, HU6 $7 \mathrm{RX}, \mathrm{UK}$

Tel.: $+44(0) 1482466656$

E-mail address: i.pires@hull.ac.uk 


\begin{abstract}
Ethnopharmacological relevance: The past few years have witnessed an increasing interest in essential oils (EOs) as potential therapeutic agents against a wide variety of pathologies, including cancer. EOs extracted from Ridolfia segetum (L.) Moris (R. segetum) are a clear example of a phytoproduct with therapeutic applications, as it is widely used in traditional medicine due to its antioxidant and anti-inflammatory properties, and these properties were already validated by previous studies. Although, it is well established that inflammation is a key hallmark of cancer, with a key role promoting tumorigenesis, and being chronic inflammation often associated with tumorigenic processes, there are no previous studies regarding the assessment of the antitumoural potential of $R$. segetum EOs.
\end{abstract}

Aim of the study: The present study intends to be the first to evaluate the antitumoural proprieties of $R$. segetum EO phytoproducts in cancer cell models.

Materials and Methods: For this, R. segetum EOs were extracted from plants collected at either flowering (RS_Fl) or fruiting (RS_Fr) stage. The impact on proliferation and viability of treatment with $R$. segetum EO extracts was assessed using in vitro 2D and 3D models.

Results: Both R. segetum EOs presented effective antiproliferative/viability effects, evidence noted by low $\mathrm{IC}_{50}$ values in $2 \mathrm{D}$ models, and significant reduction of spheroid size in $3 \mathrm{D}$ in vitro models. Mechanistically, treatment with $R$. segetum EOs was associated with an altered G1 (associated with p21 stabilisation), and subsequent induction of apoptosis.

Conclusions: Overall, these results indicate that $R$. segetum EOs have potential as suitable antitumoural therapeutic agents.

Keywords: Antitumour activity, Essential oil, Natural products, Ridolfia segetum

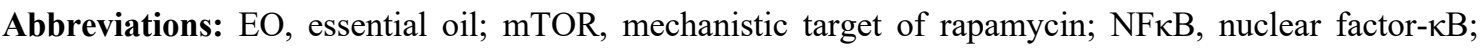
PARP, poly ADP ribose polymerase; R. segetum, Ridolfia segetum (L.) Moris; DMEM, Dulbecco's Modified Eagle's Medium; FBS, fetal bovine serum; IC 50 , half-maximal inhibitory concentration; RS_Fl, Ridolfia segetum (flowering stage); RS_Fr, Ridolfia segetum (fruiting stage).
} 


\section{Introduction}

Cancer is associated with 8.7 million deaths per year, being the second leading cause of death worldwide (Nagai and Kim, 2017). Cancer is a complex group of pathologies, which is developed through various genetic and epigenetic alterations, leading to uncontrolled cell proliferation and cell invasiveness, resistance to apoptosis, cell cycle dysregulation, and angiogenesis, amongst other (Roos et al., 2016; Sever and Brugge, 2015). Therefore, there is a continuous need for novel, effecting, antitumoural therapeutic strategies. An increasing body of research has shown that plants and their bioactive compounds present attractive therapeutic features, making plant-derived products promising therapeutic agents against cancer (Amin et al., 2015; Dhifi et al., 2016). For millennia, plants and their derived compounds have been exploited for their therapeutic features against different illnesses. Currently, some phytoproducts obtained from aromatic plants, namely essential oils (EOs), have raised high interest due to their attractive biological characteristics, such as anti-inflammatory, antiproliferative, and antioxidant activity (Blowman et al., 2018; Coté et al., 2017; do Nascimento et al., 2018; Sitarek et al., 2017). The antiproliferative and antitumour activity exhibited by many EOs is a current topic of interest in the field. In reported studies, EOs target key pathways involved in cell death and proliferation in cancer cells, including suppression of mTOR

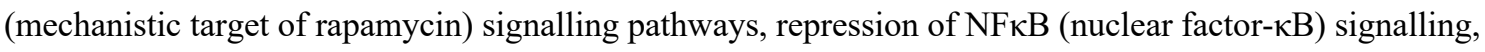
or promote caspase 3 activity and PARP (poly ADP ribose polymerase) cleavage (Blowman et al., 2018; Park et al., 2011).

Ridolfia segetum (L.) Moris (R. segetum) belongs to Apiaceae family, being widely distributed along the Mediterranean region (Cabral et al., 2015; Jabrane et al., 2010; Jannet and Mighri, 2007). This plant is already used in traditional medicine to treat gastric acidity and digestive problems, as well as, to prevent infections on the respiratory tract (Bicchi et al., 2009; Cabral et al., 2015; Jabrane et al., 2010).

Previous studies acknowledged two types of EOs extracted from $R$. segetum, with a distinct chemical composition, from samples collected from different regions and/or seasons. Therefore, an EO was identified, mainly constituted by monoterpenes hydrocarbons, like $\alpha$-phellandrene and terpinolene, which is distinguishable from those containing myristicin and dillapiole (phenylpropanoids) as major components (Cabral et al., 2015; Jabrane et al., 2010, 2009; Jannet and Mighri, 2007; Palá-Paúl et al., 2005). It was previously published, by various studies, the therapeutic potential of $R$. segetum EO as an antiinflammatory, antioxidant, and antiviral agent (Bicchi et al., 2009; Cabral et al., 2015; Jabrane et al., 2010; Marongiu et al., 2007). It is well established that inflammation is a key hallmark of cancer and has a key 
role in promoting tumorigenesis, being chronic inflammation often associated with tumorigenic processes (Hanahan and Weinberg, 2011). Chronic inflammatory processes affect all stages of tumour development as well as therapy (Elinav et al., 2013). In addition to tumour initiation, inflammation plays a decisive role in tumour promotion, malignant conversion, and metastatic dissemination (Todoric et al., 2016). So, we have to look into the correlation between inflammation and cancer. At present, cancer biology is constantly shifting from a "cancer cell centric" view to a more inclusive concept, placing cancer cells within a network of stromal cells that are comprised of fibroblasts and vascular cells and inflammatory immune cells that all together form the tumour microenvironment (Greten and Grivennikov, 2019). Bearing in mind the high anti-inflammatory potential of the EO of this species and the fact that its antitumoural activity remains unexplored, the current study is the first aiming to evaluate the antitumoural activity of R. segetum EO phytoproducts, through the assessment of the antiproliferative and cytotoxic activity of its EOs in 2D and $3 \mathrm{D}$ in vitro cancer models, as well as, the potential mechanisms of action.

\section{Material and Methods}

\subsection{Plant materials}

R. segetum was collected in Rabaçal (Portugal), during the flowering and fruiting stage. Voucher specimens were identified by a plant taxonomist (Célia Cabral) and deposited, under the numbers C. Cabral 12012 and C. Cabral 24012, in the Herbarium of Medicinal Plants, Faculty of Pharmacy, University of Coimbra, Portugal.

\subsection{Essential oil extraction and characterization}

Essential oils were extracted according to the protocol of the European Pharmacopeia (Council of Europe, 1997). It was performed by hydrodistillation for 3 hours using a Clevenger-type apparatus. The isolated EOs were kept at $4^{\circ} \mathrm{C}$ in appropriate dark glass vials. EOs chemical characterization was performed as described in Cabral et al. (Cabral et al., 2015).

\subsection{Cell culture}

RKO (colorectal cancer), MCF7 (breast cancer), and HEK293-T (immortalised human embryonic kidney fibroblasts) cell lines were cultured in Dulbecco's Modified Eagle's Medium (DMEM) (Biowest) 
supplemented with 10\% heat-inactivated fetal bovine serum (FBS) (Gibco), 1\% sodium pyruvate, and 1\% of penicillin-streptomycin (Biowest). Cells were maintained at $37^{\circ} \mathrm{C}$ under a humidified atmosphere of $5 \%$ $\mathrm{CO}_{2}$. Cells were purchased from authenticated sources (ATCC and ECACC) and were regularly tested negative for mycoplasma. The RKO and MCF7 cancer cell lines were chosen as they represent two of the most common tumour types, and are well established cell lines for this type of study, having been used for similar in vitro studies for evaluation of therapeutic response, as $2 \mathrm{D}$ models (RKO and MCF7) and 3D models (MCF7) (Beeby et al., 2020; Cazares-Körner et al., 2013; Pires et al., 2012).

\subsubsection{D spheroid models}

MCF7 3D spheroid models were developed using the liquid overlay method, as noted before (Beeby et al., 2020). Briefly, the growing monolayers were detached and seeded at a density of $2 \times 10^{4}$ cells per well in ultra-low adherence round-bottomed 96-well plates (Costar). At least 12 spheroids were formed per condition. Spheroids were treated for a total duration of 14-day, with media being replaced every 2 days. Spheroid images were obtained using a GelCount instrument (Oxford Optronix) every 2-3 days, and spheroid size was measured using the ImageJ software $(\mathrm{NIH})$.

\subsection{Evaluation of cell viability and spheroid growth inhibition}

Cell viability was assessed by measuring the metabolic activity of cells, using CellTiter 96® AQueous One Solution Cell Proliferation Assay (MTS) (Promega), following the manufacturer's protocol and as previously described (Beeby et al., 2020). In brief, HEK293-T, MCF7, and RKO cell lines were seeded at a density $2 \times 10^{4}, 1 \times 10^{4}$, and $5 \times 10^{3}$ cells per well into a 96 -well plate, respectively. After 24 hours, cells were treated with a range of $R$. segetum EOs from the flowering (RS_Fl) and fruiting (RS_Fr) stage or $\alpha$ phellandrene ( $>90 \%$, British Drug Houses, Lda) concentrations diluted in DMSO and media 1:10 (from 1 $\mu \mathrm{L} / \mathrm{mL}$ ). Doxorubicin (Sigma) diluted in media 1:10 (from $2 \mu \mathrm{M}$ ) was used as a positive control, and DMSO (vehicle) was used as a negative control. Cells were then maintained at $37^{\circ} \mathrm{C}$ and $5 \% \mathrm{CO}_{2}$ for 72 hours. Medium was removed from each well and replaced with fresh cell culture medium and MTS reagent solution. Cells were further incubated at $37^{\circ} \mathrm{C}$ and $5 \% \mathrm{CO}_{2}$. After 4 hours, the absorbance of the plate was read at $490 \mathrm{~nm}$ in a microplate reader (Bioteck ELx800, USA). Growth inhibition was evaluated as the percentage of viable cells in relation to control (vehicle-incubated cells). Half-maximal inhibitory 
concentration $\left(\mathrm{IC}_{50}\right)$ values were further calculated using GraphPad Prism Software version 8 (GraphPad Software, Inc.).

\subsection{Cell lysate preparation and western Blot}

To evaluate the expression of cell cycle and apoptosis markers, whole-cell lysates from MCF7 treated cells protein extracts were prepared as described elsewhere (Poujade et al., 2018). Briefly, cell lysates were resuspended with UTB lysis buffer (9M Urea, $75 \mathrm{mM}$ Tris- $\mathrm{HCl} \mathrm{pH} 7.5$ and $0.15 \mathrm{M} \beta$-mercaptoethanol). Lysates were sonicated for 5 minutes and centrifuged at $500 \mathrm{~g}$ for $15 \mathrm{~min}$ at $4^{\circ} \mathrm{C}$ and the resulting supernatant was transferred to a tube. Protein concentration was determined using a NanoDrop spectrophotometer software. $50 \mu \mathrm{g}$ of protein were separated using SDS-PAGE and protein expression was processed by western blot. The antibodies used for western blot were anti-p53 DO1 (sc-126, Santa Cruz Biotechnology), anti-p21 (2946, Cell Signalling Technology), and anti-PARP (9542, Cell Signalling Technology). Anti $\beta$-actin (sc-69879, Santa Cruz Biotechnology) was used as loading control. Detection was performed using the Fluorescent Imager ChemiDoc system and the Imager Lab software (Biorad). Densitometric analysis of band intensity was performed using ImageJ software (NIH) (Schneider et al., 2012). Fold changes were determined relative to the $\beta$-actin loading control bands, with vehicle only as the control samples.

\subsection{Fluorescence-activated cell sorting (FACS) cell cycle analysis}

FACS analysis was performed as previously described by Pires et al. (Pires et al., 2010). Briefly, MCF7 treated cells were detached, centrifuged, and the resulting cell pellet was resuspended in $1 \mathrm{X}$ ice-cold PBS and fixed in $70 \%$ ethanol and stored at $-20^{\circ} \mathrm{C}$ for at least 24 hours. For staining the samples, cells were washed with PBS and incubated in a $10 \mu \mathrm{g} / \mathrm{mL}$ PI and $10 \mu \mathrm{g} / \mathrm{mL}$ RNase solution in 1 X PBS and analysed using a FACS Calibur analyzer (BD Biosciences). Sample data were further analysed using the ModFIT software (Verity Software House).

\subsection{Statistical analysis}

At least three independent biological repeats were performed per experiment, with at least three experimental replicates included for MTS assays and spheroid growth inhibition assays. Results are 
expressed in terms of mean \pm SD. Statistical significance was determined by Student's t-test (one variable) or 2-way ANOVA with Tukey post hoc multiple comparison test (two variables). The differences between the means were considered significant for values of $* \mathrm{p}<0.05 ; * * \mathrm{p}<0.01$; $* * * \mathrm{p}<0.001$; and $* * * * \mathrm{p}<0.0001$. The statistical tests were performed using GraphPad Prism, version 8 (GraphPad Software, San Diego, CA, USA).

\section{Results}

\subsection{Ridolfia segetum Essential oil composition}

Chemical composition and extraction yield of both RS_Fl and RS_Fr EOs were previously determined and published in Cabral et al. (Cabral et al., 2015). Both EOs are rich in monoterpenes (95.2\% vs 85\%, respectively). The essential oil extracs main compounds are: $\alpha$-phellandrene (63.3 vs 53\%), terpinolene (11.9 vs 8.6), ß-phellandrene (6.0 vs 5.5), and dillapiol (1.9 vs 8.0\%). The main compounds from both RS_Fl and RS_Fr EOs are summarized in Table 1, more details can be found in Cabral et al 2015.

Table 1. Main chemical compounds of $R$. segetum essential oils from both flowering and fruiting stages (Cabral et al., 2015).

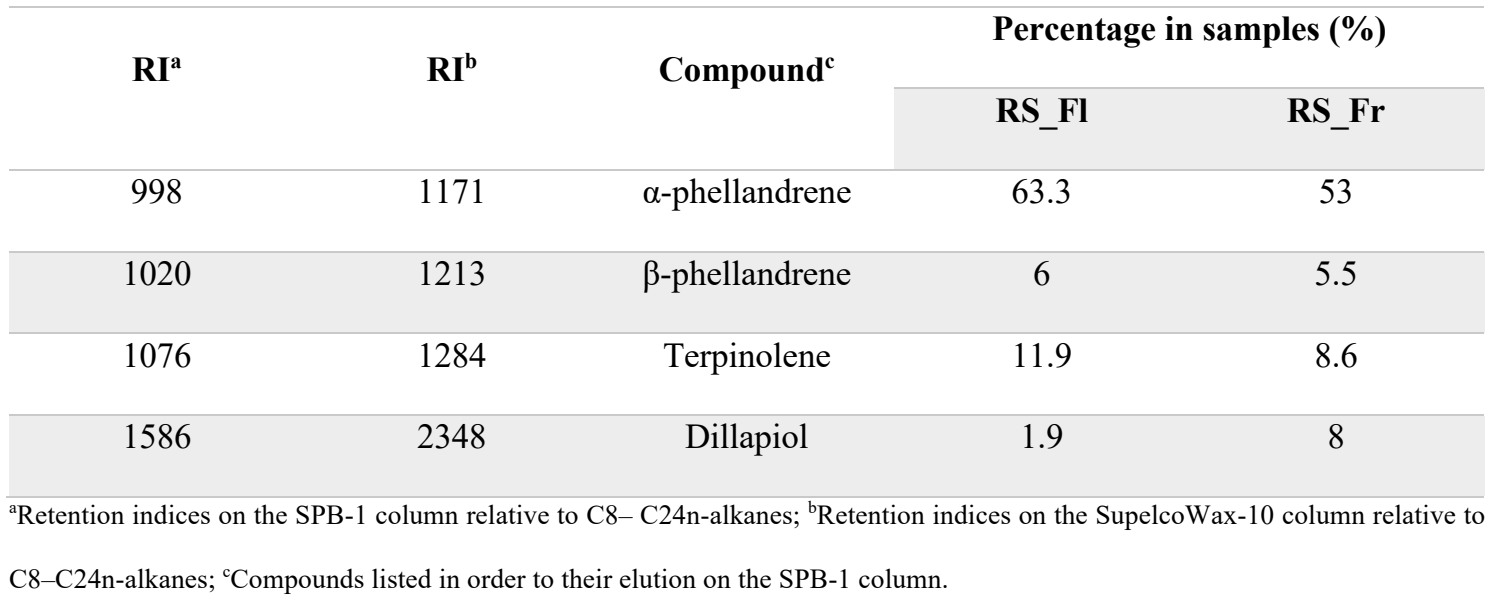




\subsection{Antiproliferative effect of Ridolfia segetum essential oil}

The antitumoural activity of RS_Fl (extracted from flowering R. Segetum) and RS_Fr (extracted from fruiting R. Segetum) EOs was evaluated. This was done alongside an evaluation of the therapeutic activity of $\alpha$-phellandrene, as this is the main compound of both EOs, as we have previously shown (Cabral et al., 2015). Firstly, the antiproliferative activity of $R$. segetum EOs was assessed comparing to $\alpha$-phellandrene alone and doxorubicin using the 2D MTS assay. The impact of EOs treatment in the viability/proliferation was determined in both MCF7 and RKO cancer cell lines (Figure 1A and B) as well as non-cancer cell line HEK293-T (Figure 1C), and included the calculation of the relevant IC50 values (concentration needed to inhibit proliferation by $50 \%$ ) (Figure 1D). The data showed that EOs from both stages induced a significant decrease in cell viability for the cancer cell lines, comparable to that exhibited by conventional anticancer agent Doxorubicin (Figure 2). IC50 values were lower for RS_Fl EO, although the half-inhibitory concentration values did not significantly differ from the RS_Fr EO (Figure 1D). This antiproliferative effect by $R$. segetum EOs was not observed in the non-cancer cell line (HEK293-T cells), with IC50 nearly 20 times higher than those observed for MCF7 and RKO cells (Figure 1C and D). Interestingly, treatment with $\alpha$-phellandrene did not significantly affect cell viability for both cell lines (Figure 1A and B). Therefore, RS_Fl and RS_Fr EOs composition (a mixture of monoterpenes) seem to be responsible for EOs antiproliferative activity against cancer cells, once $\alpha$-phellandrene alone did not promote any therapeutic effect. 
A

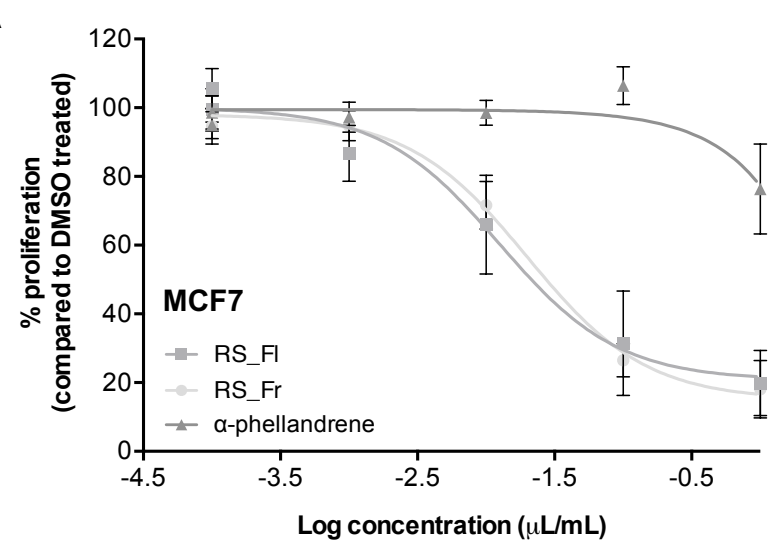

C

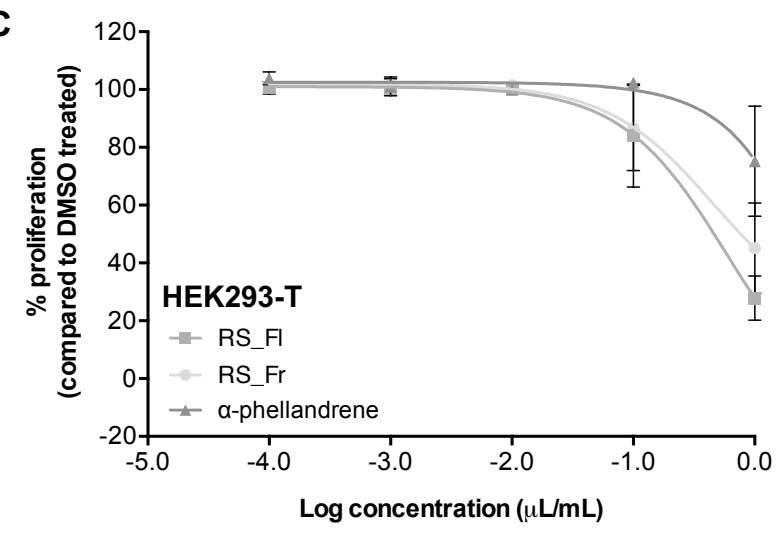

B

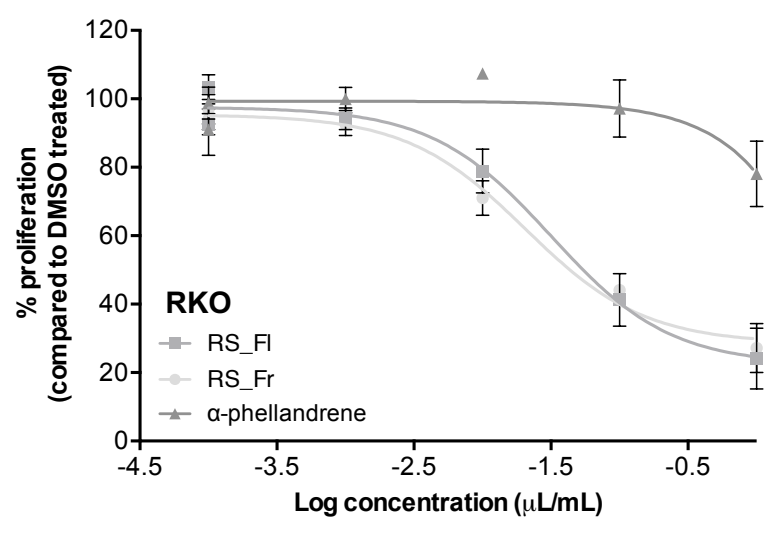

D

\begin{tabular}{|l|c|c|c|}
\hline \multirow{2}{*}{ Species } & \multicolumn{3}{|c|}{ IC $_{\mathbf{5 0}}(\boldsymbol{\mu L} / \mathrm{mL})$} \\
\cline { 2 - 4 } & MCF7 & RKO & HEK293-T \\
\hline$R S \_F I$ & 0.021 & 0.025 & 0.45 \\
\hline$R S-F r$ & 0.025 & 0.031 & 0.6 \\
\hline a-phellandrene & ND & ND & ND \\
\hline
\end{tabular}

Figure 1. Cytotoxic effect of essential oils from Ridolfia segetum and its component $\alpha$-phellandrene.

The cytotoxic effect of essential oils from Ridolfia segetum (flowering (RS_Fl) and fruiting (RS_Fr)) or the component $\alpha$-phellandrene was evaluated against cancer cell lines MCF7 (A) and RKO (B), as well as noncancer cell line HEK293-T (C). Cells were seeded at a density of $1 \times 10^{4}, 5 \times 10^{3}$, and $2 \times 10^{4}$ cells per well in a 96-well plate, respectively. Cells were then treated with a dose range of compounds concentrations or vehicle control (DMSO) for $72 \mathrm{~h}$, and subsequently an MTS assay was performed. Cell viability is represented of three independent experiments. Plots represent the mean \pm SD of three independent biological repeats, each with three experimental repeats (wells) per condition, and is expressed as percentage survival of control. IC 50 values for each EO mixtures per cell line is noted in (D). ND: value not determined as IC $>50$. 


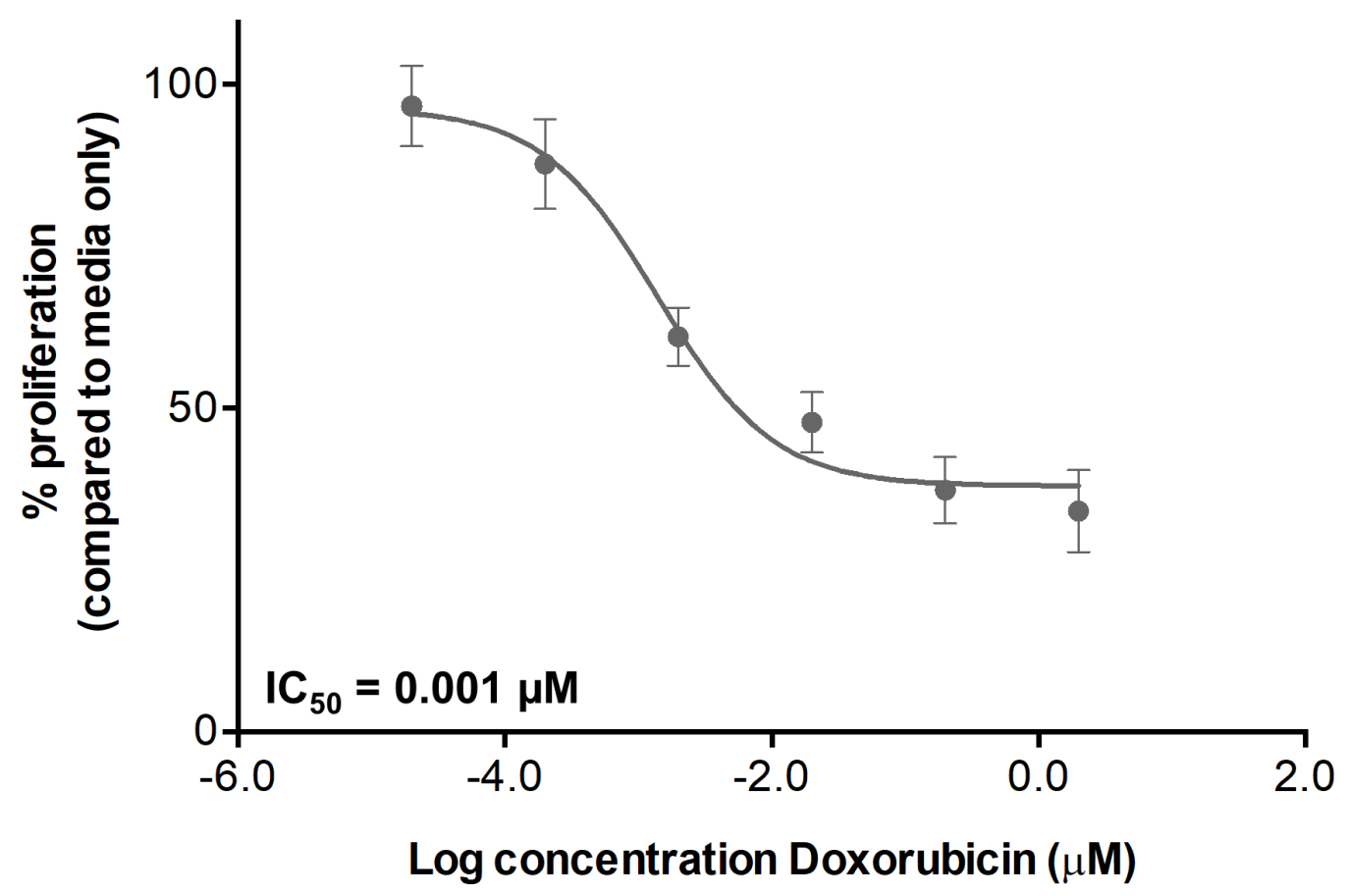

Figure 2. Cytotoxic effect of doxorubicin against MCF7 cells.

Cells were treated with a range of doxorubicin concentrations (from $2 \mu \mathrm{M}$ ) for 72 hours and an MTS assay was performed. Cell viability was expressed as percentage survival of control $(n=3$ independent experiments). $\mathrm{IC}_{50}$ value represents the average of three independent experiments, each with three experimental repeats (wells) per condition. 


\subsection{Antitumour activity of Ridolfia segetum essential oil against 3D spheroid models}

As shown in the previous section, both EOs from RS_Fl and RS_Fr induced a strong decrease in cell proliferation/viability in 2D cancer models. To evaluate the efficacy of these EOs in models better reflecting the tumour microenvironment, 3D spheroid models were used, as these are well-established in vitro models to study novel therapeutic approaches against cancer, since these models simulate tumour microenvironment in vitro (Zanoni et al., 2016). MCF7 spheroids were formed and treated during 14 days with RS_Fl and RS_Fr EOs and $\alpha$-phellandrene, with the spheroid diameter measured over time (Figure 3). Treatment with R. segetum EOs induced a significant reduction of the spheroids' diameter over time, with the most pronounced effect noted in spheroids treated with RS_Fr EO. Treatment with both EOs from RS_Fl and RS_Fr induced a decrease in spheroid volume from day 7 (Figure 3A). Complete spheroid disaggregation was observed for RS_Fr EO by day 12, whilst, even at day 14, RS_Fl EO promoted a significant inhibition of spheroid growth but not complete disaggregation (Figure 3B and C). Treatment with $\alpha$-phellandrene also led to a significant decrease in spheroid diameter when compared to the vehicle control, but still also significantly larger than the R. segetum EOs treated spheroids (Figure 3D). However, although the $\alpha$-phellandrene-treated spheroids were smaller than the DMSO control from day 7, these did not appear disaggregated, and the diameter was not significantly different when comparing day 0 with any other timepoints (Figure 3C). This indicates that, unlike the RS_Fl and RS_Fr EOs, $\alpha$-phellandrene treatment might have a predominantly cytostatic, rather than cytotoxic, effect. Therefore, the antiproliferative activity observed after spheroids treatment with RS_Fl and RS_Fr EOs support the previous results, once the cytotoxic effect was not due to $\alpha$-phellandrene alone (mostly cytostatic effect) but probably to the mixture of monoterpenes in EOs composition. 
A

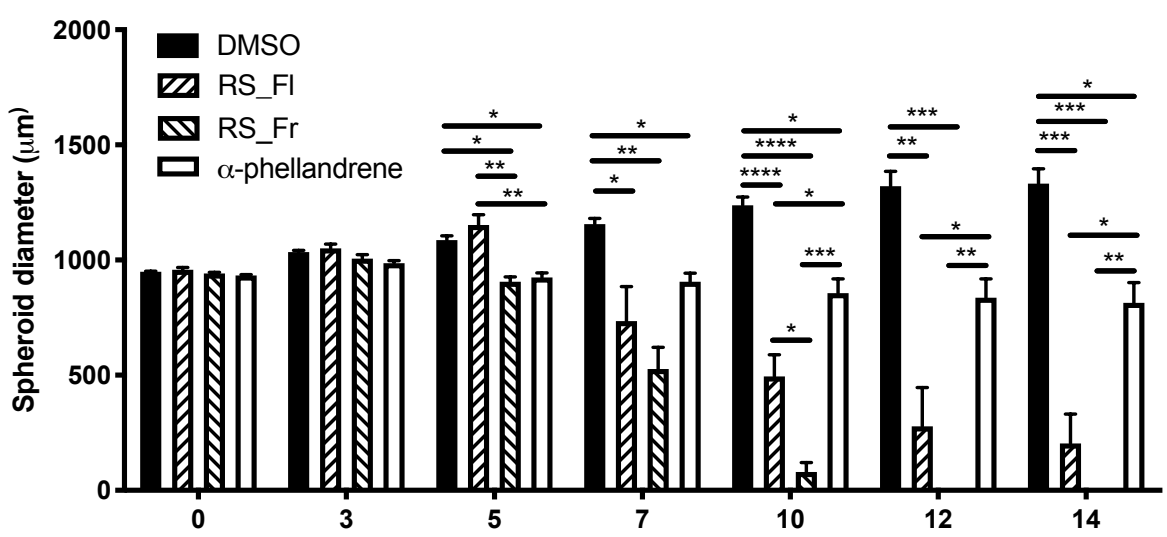

B

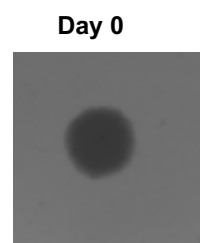

Day 7

Day 14
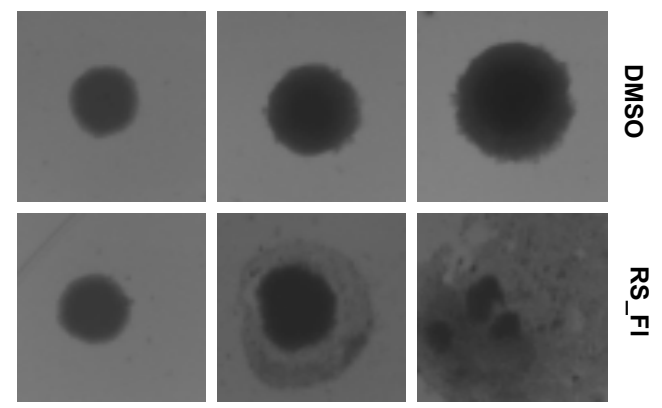

C

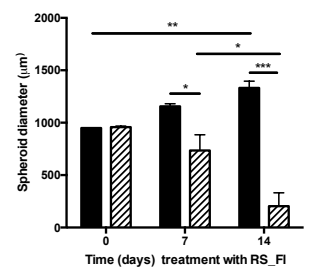

D

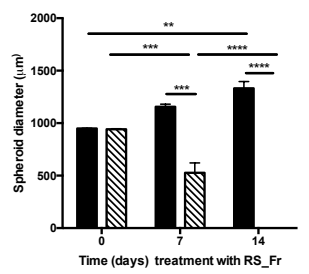

E

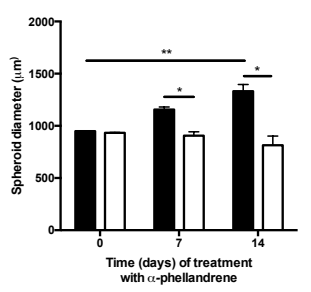

Figure 3. Impact of treatment with Ridolfia segetum essential oils on in vitro 3D cancer models.

MCF7 3D spheroids were treated with either vehicle-only control (1 $\mu \mathrm{L} / \mathrm{ml}$ DMSO in media), or $1 \mu \mathrm{L} / \mathrm{ml}$ RS_Fl, or RS_Fr EOs, $\alpha$-phellandrene in media. Treatment was maintained for 14 days, with media being refreshed every 2-3 days. Spheroids were imaged every 2-3 days and spheroid volume was assessed. (A) Histogram representing the mean spheroid volume after treatment with both RS_Fl and RS_Fr EOs and $\alpha-$ phellandrene alone; (B) Representative images of treated spheroids at days 0, 7, and 14; (C) Assessment of mean spheroid diameter after treatment with RS_Fl EO at days 0, 7, and 14; (D) Assessment of mean spheroid diameter after treatment with RS_Fr EO at days 0, 7, and 14; (E) Assessment of mean spheroid diameter after treatment with $\alpha$-phellandrene alone at days 0,7 , and 14 . Histograms represent mean $\pm \mathrm{SD}$, being representative of at least three independent experiments, each with three experimental repeats (wells) per condition. ${ }^{*} p<0.05 ; * * p<0.01 ;{ }^{* * *} p<0.001 ; * * * * p<0.0001$. 


\subsection{Antitumour mechanism of action of Ridolfia segetum essential oil}

Next, the mechanism of action of the $R$. segetum EOs was evaluated by assessing the impact of treatment with both EOs in cell-cycle regulation and apoptosis signalling pathways (Figure 4). MCF7 cells were treated with $1 \mu \mathrm{L} / \mathrm{mL}$ RS_Fl and RS_Fr EOs for 24 (cell-cycle and apoptosis signalling pathways analysis) and 48 hours (cell-cycle analysis). Regarding cell-cycle progression, a significant decrease in cell population in G1 was observed for both EOs at 48h (Figure 4A). Furthermore, treatment with RS_Fl EO induced a significant enhance in the percentage of cells in sub-G1 cell-cycle phase when compared with the condition treated with DMSO (vehicle) at both $24 \mathrm{~h}$ and $48 \mathrm{~h}$, whereas a significant increase in sub-G1 was also observed for RS_Fr EO treated cells (Figure 4A).

In order to evaluate the mechanism underpinning the cell-cycle phenotypes, the levels of p53 and p21 key cell-cycle regulatory factors were evaluated by western blot (Figure 4B). Treatment with both RS_Fl and RS_Fr EOs led to increased levels of p21, but not p53 (Figure 4B).

Finally, as an increase of cells in sub-G1 phase is associated with a potential increase in apoptosis, a western blot was performed to analyse the levels of total (tPARP) and cleaved (cPARP) PARP (the latter being a marker of apoptosis downstream of caspase activation) (Figure 4B). 24 hours after treatment with both RS_Fl and RS_Fr EOs, a decrease in TPARP levels relative to the control condition was observed, with a concomitant increase in cPARP in both treated conditions. Therefore, these data indicate that the antitumour mechanism for RS_Fl and RS_Fr EOs is occurring via induction of apoptosis, associated with dysregulation of cell cycle progression. 
A

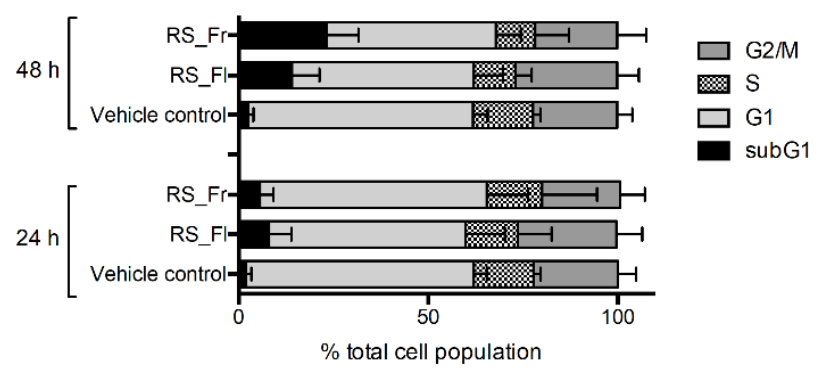

\begin{tabular}{|c|c|c|c|c|c|}
\hline & & subG1 & G1 & $s$ & G2/M \\
\hline \multirow{2}{*}{$24 \mathrm{~h}$} & $\begin{array}{c}\text { Vehicle only vs } \\
\text { RS_FI }\end{array}$ & ${ }^{*} p<0.05$ & $p>0.05$ & $p>0.05$ & $p>0.05$ \\
\hline & $\begin{array}{c}\text { Vehicle only vs } \\
\text { RS_Fr }\end{array}$ & $p>0.05$ & $p>0.05$ & $p>0.05$ & $p>0.05$ \\
\hline \multirow{2}{*}{$48 \mathrm{~h}$} & $\begin{array}{c}\text { Vehicle only vs } \\
\text { RS_FI }\end{array}$ & ${ }^{*} p<0.05$ & ${ }^{* \star} p<0.01$ & $p>0.05$ & $p>0.05$ \\
\hline & $\begin{array}{c}\text { Vehicle only vs } \\
\text { RS_Fr }\end{array}$ & ${ }^{* *} p<0.01$ & ${ }^{* *} p<0.01$ & $p>0.05$ & $p>0.05$ \\
\hline
\end{tabular}

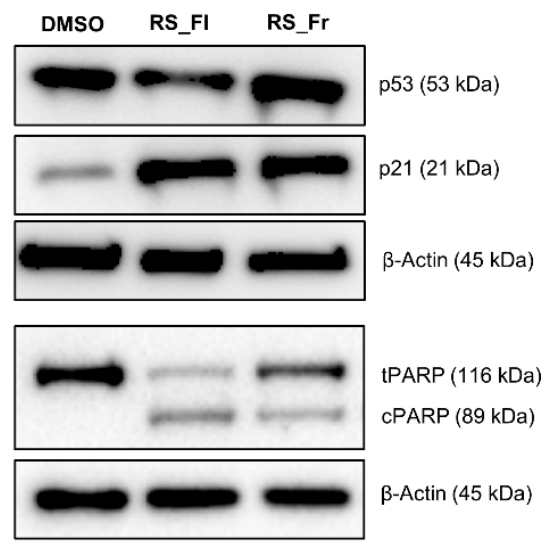

Figure 4. Impact of treatment with Ridolfia segetum essential oils (RS_Fl and RS_Fr) on cell cycle regulation cell survival and cell death signaling.

MCF7 cells were treated with either the vehicle-only control $(1 \mu \mathrm{L} / \mathrm{mL}$ DMSO in media) or with $1 \mu \mathrm{L} / \mathrm{mL}$ RS_Fl and RS_Fr EOs in media. (A) After 24 and 48 hours, samples were analysed by flow cytometry for DNA content. Proportion of cells in subG1, G1, S, and G2/M are presented as percentages of total cell population. Plots represent mean \pm SD for three independent experiments and the statistical significance corresponds to the differences between means of vehicle control vs RS_Fl EO or RS_Fr EO, per timepoint and cell cycle phase. P values are noted in the table. (B) After 24 hours, p53, p21, cleaved PARP (cPARP), and total PARP (tPARP) levels were analysed by western blotting. $\beta$-actin was used as loading control. Data show representative blots of three independent experiments. Quantification of band intensity and subsequent statistical analysis is available on Supplementary Figure 1. 


\section{Discussion}

Over the years, various studies already explored some therapeutic features of the EO obtained from $R$. segetum, focusing on its capability to act as a viable and efficient anti-inflammatory, antioxidant, antibacterial, and antiviral agent (Bicchi et al., 2009; Cabral et al., 2015; Jabrane et al., 2010, 2009). In this work, the yet unexplored antitumour potential of RS_Fl and RS_Fr EOs was assessed.

In this regard, as previously published by Cabral et al., these EOs were obtained from RS_Fl and RS_Fr stage with a considerable high yield (1.6\% vs $1.9 \%$, respectively), in which the main compounds obtained were monoterpenes ( $95.2 \%$ vs $85 \%$, respectively), especially $\alpha$-phellandrene (63.3 vs 53\%, respectively) (Cabral et al., 2015). These data are in accordance with the described composition in the literature for this type of EO (Jabrane et al., 2009; Marongiu et al., 2007; Palá-Paúl et al., 2005). Furthermore, some researchers have stated that the presence of monoterpenes, including $\alpha$-phellandrene, in EOs composition has been widely associated with antitumour activity (Lin et al., 2014b, 2014a; Sobral et al., 2014). Concerning this, the referred association between monoterpenes and the antitumour activity attributed to EOs was considered in the development of this work, carrying a high interest to study the antitumour activity of $R$. segetum EOs.

After cancer cells treatment with both RS_Fl and RS_Fr EOs, a therapeutic effect was observed by the significant decrease on cell viability and proliferative capability of cancer cells (Figure 1). Furthermore, this antitumour effect observed for RS_Fl and RS_Fr EOs presents a similar profile to the observed by doxorubicin treatment, suggesting that EOs from both stages act as cytotoxic agents (Figure 1 and 2). Moreover, this therapeutic effect of RS_Fl and RS_Fr EOs was also sustained by a significant reduction of the spheroid growth (Figure 3). Surprisingly, treatment with $\alpha$-phellandrene alone did not induce any significant cytotoxic effect, which was not expected since some works report the antitumour effect promoted by this monoterpene hydrocarbon (Lin et al., 2014b, 2014a). According to the results obtained, $\alpha$-phellandrene did not disturb cancer cell viability, as well as, did not promote a reduction of the spheroid size over time in $3 \mathrm{D}$ in vitro cancer models, suggesting a cytostatic rather than cytotoxic activity (Figure 1 and Figure 3). Thus, the antitumour effect observed in cells treated with RS_Fl and RS_Fr EOs may not be attributed to $\alpha$-phellandrene alone, but most probably to the complex monoterpene mixture. Additionally, the striking antiproliferative effect exerted by RS_Fl and RS_Fr EOs against cancer cell lines was not observed in a non-cancer cell line (HEK-293T), using the same concentrations (Figure 1). This indicates that treatment with both RS_Fl and RS_Fr EOs, and its strong cytotoxic effect in cancer cells but not in 
non-cancer cells, promote a large therapeutic window and reduced non-cancer toxicity for any future potential anticancer agents derived from these EOs. However, this will need further study with more complex in vivo models.

Furthermore, RS_Fl and RS_Fr EOs antiproliferative activity may be explained by its composition (mixture of monoterpenes), once treatment with monoterpenes or EOs composed mainly by monoterpenes induces a decrease on cell proliferation. An example is the study of Aydin et al., in which they showed the antioxidant and antitumour potential of terpinolene (monoterpene compound) against neuroblastoma cells by decreasing the proliferative capability of cancer cells (Aydin et al., 2013). Following, preclinic studies performed by Mare et al. exposed the antitumour potential of monoterpenes collected from algae species against breast cancer cells (De La Mare et al., 2012). In this work, monoterpenes were widely related to apoptosis inducement and inhibition of proliferative capability. Various other studies also have established a relation between the antitumour potential exhibited by different plant species and the presence of monoterpenes in EOs composition, in which the antitumour activity is attributed to different mechanisms such as cell cycle arrest, inhibition of cell proliferation, and induction of apoptosis by caspase-dependent pathways (Al-Dabbagh et al., 2019, 2018; Gautam et al., 2016; Hsieh et al., 2010; Sigurdsson et al., 2005). The obtained results showed that treatment with both RS_Fl and RS_Fr EOs affected cell cycle progression with a significant increase of cells in sub-G1 phase and dysregulation of G1 progression (Figure 4A). Furthermore, the levels of p53 did not differ from control condition (DMSO), which can indicate that the increase of cyclin-dependent kinase inhibitor p21 protein levels was not p53-dependent and, consequently, that cell death induction was not associated with DNA damage induction in the presence of the EOs (Figure 4A). Furthermore, an increase in PARP cleavage, a well-established marker of apoptosis, was observed, showing that these EOs induce apoptosis in cancer cells (Figure 4B), indicating that the antitumour mechanism of action of RS_Fl and RS_Fr EOs is mediated via caspase-dependent apoptosis (VizettoDuarte et al., 2016). Future work perspectives should include a deepener evaluation of apoptosis induction mechanisms in vitro, as well as, study possible therapeutic alternatives, like conjugation with chemotherapeutic approaches, both in vitro and in vivo.

\section{Conclusion}

In summary, this work was able to fulfil the lack of information about the therapeutic potential of RS_Fl and RS_Fr EOs against cancer cells. Moreover, the results observed in this study suggest that both EOs 
present a strong antitumour potential with a higher capability to inhibit proliferation and induce apoptosis, being promising compounds to be used in future antitumour therapeutic strategies.

\section{Acknowledgments}

This work was supported through HEFCE funding provided by the University of Hull (IMP and EB), by Fundação para a Ciência e Tecnologia Strategic Projects UID/MAR/04292/2013 grant to MARE; UID/NEU/04539/2013 and UID/NEU/04539/2019 grant to CNC.IBILI, UIDB/04539/2020 and UIDP/04539/2020 grant to CIBB; and by the Integrated Programme of SR\&TD "SmartBioR" (reference Centro-01-0145-FEDER-000018), co-funded by Centro 2020 program, Portugal 2020, European Union, through the European Regional Development Fund.

\section{Conflicts of interest}

The author(s) declare(s) that there is no conflict of interest regarding the publication of this paper.

\section{List of the authors and respective contributions}

Experiments were carried out by EB, IMP, and CC. Data analysis was carried out by EB, CC, and IMP. IMP and CC designed the experiments. MM, CC, and IMP wrote the paper with contributions and editing by all other authors. Funding was secured by ML, CC, and IMP.

\section{References}

Al-Dabbagh, B., Elhaty, I.A., Al Hrout, A., Al Sakkaf, R., El-Awady, R., Ashraf, S.S., Amin, A., 2018. Antioxidant and anticancer activities of Trigonella foenum-graecum, Cassia acutifolia and Rhazya stricta. BMC Complement. Altern. Med. 18, 240. https://doi.org/10.1186/s12906-018-2285-7

Al-Dabbagh, B., Elhaty, I.A., Elhaw, M., Murali, C., Al Mansoori, A., Awad, B., Amin, A., 2019. Antioxidant and anticancer activities of chamomile (Matricaria recutita L.). BMC Res. Notes 12, 3. https://doi.org/10.1186/s13104-018-3960-y

Amin, A., Bajbouj, K., Koch, A., Gandesiri, M., Schneider-Stock, R., 2015. Defective Autophagosome Formation in p53-Null Colorectal Cancer Reinforces Crocin-Induced Apoptosis. Int. J. Mol. Sci. . https://doi.org/10.3390/ijms16011544 
Aydin, E., Türkez, H., Taşdemir, Ş., 2013. Anticancer and antioxidant properties of terpinolene in rat brain cells. Arh. Hig. Rada Toksikol. 64, 415-424. https://doi.org/10.2478/10004-1254-64-20132365

Beeby, E., Magalhães, M., Poças, J., Collins, T., Lemos, M.F.L., Barros, L., Ferreira, I.C.F.R., Cabral, C., Pires, I.M., 2020. Secondary metabolites (essential oils) from sand-dune plants induce cytotoxic effects in cancer cells. J. Ethnopharmacol. 258, 112803. https://doi.org/10.1016/j.jep.2020.112803

Bicchi, C., Rubiolo, P., Ballero, M., Sanna, C., Matteodo, M., Esposito, F., Zinzula, L., Tramontano, E., 2009. HIV-1-inhibiting activity of the essential oil of ridolfia segetum and oenanthe crocata. Planta Med. 75, 1331-1335. https://doi.org/10.1055/s-0029-1185546

Blowman, K., Magalhães, M., Lemos, M.F.L., Cabral, C., Pires, I.M., 2018. Anticancer properties of essential oils and other natural products. Evidence-based Complement. Altern. Med. 2018.

Cabral, C., Poças, J., Gonçalves, M.J., Cavaleiro, C., Cruz, M.T., Salgueiro, L., 2015. Ridolfia segetum (L.) Moris (Apiaceae) from Portugal: A source of safe antioxidant and anti-inflammatory essential oil. Ind. Crops Prod. 65, 56-61. https://doi.org/10.1016/j.indcrop.2014.11.041

Cazares-Körner, C., Pires, I.M., Swallow, I.D., Grayer, S.C., O’Connor, L.J., Olcina, M.M., Christlieb, M., Conway, S.J., Hammond, E.M., 2013. CH-01 is a Hypoxia-Activated Prodrug That Sensitizes Cells to Hypoxia/Reoxygenation Through Inhibition of Chk1 and Aurora A. ACS Chem. Biol. 8, 1451-1459. https://doi.org/10.1021/cb4001537

Coté, H., Boucher, M.-A., Pichette, A., Legault, J., 2017. Anti-inflammatory, antioxidant, antibiotic, and cytotoxic activities of Tanacetum vulgare L. essential oil and its constituents. Medicines 4, 34 . https://doi.org/10.3390/medicines4020034

Council of Europe, 1997. European Pharmacopoeia., third ed. ed. Council of Europe, Strasbourg.

De La Mare, J.A., Lawson, J.C., Chiwakata, M.T., Beukes, D.R., Edkins, A.L., Blatch, G.L., 2012. Quinones and halogenated monoterpenes of algal origin show anti-proliferative effects against breast cancer cells in vitro. Invest. New Drugs 30, 2187-2200. https://doi.org/10.1007/s10637-0119788-0

Dhifi, W., Bellili, S., Jazi, S., Bahloul, N., Mnif, W., 2016. Essential oils' chemical characterization and investigation of some biological activities: A critical review. Medicines 3, 25. https://doi.org/10.3390/medicines3040025 do Nascimento, K.F., Moreira, F.M.F., Alencar Santos, J., Kassuya, C.A.L., Croda, J.H.R., Cardoso, 
C.A.L., Vieira, M. do C., Góis Ruiz, A.L.T., Ann Foglio, M., de Carvalho, J.E., Formagio, A.S.N., 2018. Antioxidant, anti-inflammatory, antiproliferative and antimycobacterial activities of the essential oil of Psidium guineense Sw. and spathulenol. J. Ethnopharmacol. 210, 351-358. https://doi.org/10.1016/j.jep.2017.08.030

Elinav, E., Nowarski, R., Thaiss, C.A., Hu, B., Jin, C., Flavell, R.A., 2013. Inflammation-induced cancer: crosstalk between tumours, immune cells and microorganisms. Nat. Rev. Cancer 13, 759-771. https://doi.org/10.1038/nrc3611

Gautam, L.N., Ling, T., Lang, W., Rivas, F., 2016. Anti-proliferative evaluation of monoterpene derivatives against leukemia. Eur. J. Med. Chem. 113, 75-80. https://doi.org/10.1016/j.ejmech.2016.02.034

Greten, F.R., Grivennikov, S.I., 2019. Inflammation and Cancer: Triggers, Mechanisms, and Consequences. Immunity 51, 27-41. https://doi.org/https://doi.org/10.1016/j.immuni.2019.06.025

Hanahan, D., Weinberg, R.A., 2011. Hallmarks of cancer: The next generation. Cell 144, 646-674. https://doi.org/10.1016/j.cell.2011.02.013

Hsieh, C.C., Kuo, Y.H., Kuo, C.C., Chen, L.T., Cheung, C.H.A., Chao, T.Y., Lin, C.H., Pan, W.Y., Chang, C.Y., Chien, S.C., Chen, T.W., Lung, C.C., Chang, J.Y., 2010. Chamaecypanone C, a novel skeleton microtubule inhibitor, with anticancer activity by trigger caspase 8-Fas/FasL dependent apoptotic pathway in human cancer cells. Biochem. Pharmacol. 79, 1261-1271. https://doi.org/10.1016/j.bcp.2009.12.017

Jabrane, A., Ben Jannet, H., Mastouri, M., Mighri, Z., Casanova, J., 2010. Chemical composition and in vitro evaluation of antioxidant and antibacterial activities of the root oil of Ridolfia segetum (L.) Moris from Tunisia. Nat. Prod. Res. 24, 491-499. https://doi.org/10.1080/14786410802228520

Jabrane, A., Jannet, H. Ben, Harzallah-Skhiri, F., Casanova, J., Mighri, Z., 2009. GC, GC-MS and13C NMR spectroscopy integrated analyses and in vitro antibacterial activity of ridolfia segetum essential oils from tunisia. J. Essent. Oil-Bearing Plants 12, 521-530. https://doi.org/10.1080/0972060X.2009.10643752

Jannet, H. Ben, Mighri, Z., 2007. Hydrodistillation kinetic and antibacterial effect studies of the flower essential oil from the tunisian ridolfia segetum (L.). J. Essent. Oil Res. 19, 258-261. https://doi.org/10.1080/10412905.2007.9699273

Lin, J.-J., Lu, K.-W., Ma, Y.-S., Tang, N.-Y., Wu, P.-P., Wu, C.-C., Lu, H.-F., Lin, J.-G., Chung, J.-G., 
2014a. Alpha-phellandrene, a natural active monoterpene, influences a murine WEHI-3 leukemia model in vivo by enhancing macrophague phagocytosis and natural killer cell activity. In Vivo (Brooklyn). 28, 583-588.

Lin, J.-J., Yu, C.-C., Lu, K.-W., Chang, S.-J., Yu, F.-S., Liao, C.-L., Lin, J.-G., Chung, J.-G., 2014b. $\alpha-$ Phellandrene alters expression of genes associated with DNA damage, cell cycle, and apoptosis in murine leukemia WEHI-3 cells. Anticancer Res. 34, 4161-80.

Marongiu, B., Piras, A., Porcedda, S., Tuveri, E., Maxia, A., 2007. Comparative analysis of the oil and supercritical CO2 extract of Ridolfia segetum (L.) Moris. Nat. Prod. Res. 21, 412-417. https://doi.org/10.1080/00319100600577443

Nagai, H., Kim, Y.H., 2017. Cancer prevention from the perspective of global cancer burden patterns. J. Thorac. Dis. 9, 448-451. https://doi.org/10.21037/jtd.2017.02.75

Palá-Paúl, J., Velasco-Negueruela, A., Pérez-Alonso, M.J., Vallejo, M.C.G., 2005. Volatile constituents of ridolfia segetum (1.) moris gathered in central spain: Castilla la Mancha Province. J. Essent. Oil Res. 17, 119-121. https://doi.org/10.1080/10412905.2005.9698848

Park, K.-R., Nam, D., Yun, H.-M., Lee, S.-G., Jang, H.-J., Sethi, G., Cho, S.K., Ahn, K.S., 2011. $\beta$ Caryophyllene oxide inhibits growth and induces apoptosis through the suppression of PI3K/AKT/mTOR/S6K1 pathways and ROS-mediated MAPKs activation. Cancer Lett. 312, 178188. https://doi.org/https://doi.org/10.1016/j.canlet.2011.08.001

Pires, I.M., Bencokova, Z., Milani, M., Folkes, L.K., Li, J.-L., Stratford, M.R., Harris, A.L., Hammond, E.M., 2010. Effects of Acute versus Chronic Hypoxia on DNA Damage Responses and Genomic Instability. Cancer Res. 70, 925-935. https://doi.org/10.1158/0008-5472.CAN-09-2715

Pires, I.M., Olcina, M.M., Anbalagan, S., Pollard, J.R., Reaper, P.M., Charlton, P.A., McKenna, W.G., Hammond, E.M., 2012. Targeting radiation-resistant hypoxic tumour cells through ATR inhibition. Br. J. Cancer 107, 291-299. https://doi.org/10.1038/bjc.2012.265

Poujade, F.-A., Mannion, A., Brittain, N., Theodosi, A., Beeby, E., Leszczynska, K.B., Hammond, E.M., Greenman, J., Cawthorne, C., Pires, I.M., 2018. WSB-1 regulates the metastatic potential of hormone receptor negative breast cancer. Br. J. Cancer 118, 1229-1237. https://doi.org/10.1038/s41416-018-0056-3

Roos, W.P., Thomas, A.D., Kaina, B., 2016. DNA damage and the balance between survival and death in cancer biology. Nat. Rev. Cancer 16, 20-33. https://doi.org/10.1038/nrc.2015.2 
Schneider, C.A., Rasband, W.S., Eliceiri, K.W., 2012. NIH Image to ImageJ: 25 years of image analysis. Nat. Methods 9, 671-675. https://doi.org/10.1038/nmeth.2089

Sever, R., Brugge, J.S., 2015. Signal transduction in cancer. Cold Spring Harb. Perspect. Med. 5, a006098. https://doi.org/10.1101/cshperspect.a006098

Sigurdsson, S., Ögmundsdottir, H.M., Gudbjarnason, S., 2005. The cytotoxic effect of two chemotypes of essential oils from the fruits of Angelica archangelica L. Anticancer Res. 25, 1877-1880.

Sitarek, P., Rijo, P., Garcia, C., Skała, E., Kalemba, D., Białas, A.J., Szemraj, J., Pytel, D., Toma, M., Wysokinska, H., Sliwinski, T., 2017. Antibacterial, anti-inflammatory, antioxidant, and antiproliferative properties of essential oils from hairy and normal roots of Leonurus sibiricus L. and their chemical composition. Oxid. Med. Cell. Longev. 2017, 1-12. https://doi.org/10.1155/2017/7384061

Sobral, M.V., Xavier, A.L., Lima, T.C., De Sousa, D.P., 2014. Antitumor activity of monoterpenes found in essential oils. Sci. World J. 2014. https://doi.org/10.1155/2014/953451

Todoric, J., Antonucci, L., Karin, M., 2016. Targeting Inflammation in Cancer Prevention and Therapy. Cancer Prev. Res. 9, 895 LP - 905. https://doi.org/10.1158/1940-6207.CAPR-16-0209

Vizetto-Duarte, C., Custódio, L., Gangadhar, K.N., Lago, J.H.G., Dias, C., Matos, A.M., Neng, N., Nogueira, J.M.F., Barreira, L., Albericio, F., Rauter, A.P., Varela, J., 2016. Isololiolide, a carotenoid metabolite isolated from the brown alga Cystoseira tamariscifolia, is cytotoxic and able to induce apoptosis in hepatocarcinoma cells through caspase-3 activation, decreased Bcl-2 levels, increased p53 expression and PARP cleava. Phytomedicine 23, 550-557. https://doi.org/10.1016/j.phymed.2016.02.008

Zanoni, M., Piccinini, F., Arienti, C., Zamagni, A., Santi, S., Polico, R., Bevilacqua, A., Tesei, A., 2016. 3D tumor spheroid models for in vitro therapeutic screening: a systematic approach to enhance the biological relevance of data obtained. Sci. Rep. 1-11. https://doi.org/10.1038/srep19103 